\title{
A framework for safe integration of small UAS into the NAS
}

\author{
Michael J. Logan, $\mathrm{PE}^{1}$ \\ NASA Langley Research Center, Hampton, VA \\ Geoffrey Bland ${ }^{2}$ \\ NASA GSFC/Wallops Flight Facility, Wallops Island, VA \\ and \\ Jennifer Murray ${ }^{3}$ \\ NASA Kennedy Space Center, FL
}

\begin{abstract}
This paper discusses a proposed framework for the safe integration of small unmanned aerial systems (sUAS) into the National Airspace System (NAS). The paper examines the potential uses of sUAS to build an understanding of the location and frequency of potential future flight operations based on the future applications of the sUAS systems. The paper then examines the types of systems that would be required to meet the application-level demand to determine "classes" of platforms and operations. Finally, a framework is proposed for both airworthiness and operations that attempts to balance safety with utility for these important systems.
\end{abstract}

\section{Nomenclature}

UAS = Unmanned Aircraft System

NAS $\quad=$ National Airspace System

sUAS $\quad=$ small UAS

\section{Introduction}

$\mathrm{T}$ There has been a tremendous upsurge in the desire for small, Unmanned Aerial Systems (sUAS) to be able to freely access the National Airspace System (NAS). In order to assess the need and possible impact on the NAS, it is imperative to investigate three basic questions. First, what is the demand for sUAS from an application and usage standpoint? Two, what are the potential benefits of broad sUAS access in terms of an economic benefit analysis as well as benefits to the public good? Three, what are the roadblocks to broad access from regulatory, safety, and infrastructure concerns? Finally, it is also beneficial to postulate a path forward to address the current roadblocks which will potentially open the broadest access for sUAS.

\section{Application Demand}

To determine the potential demand for sUAS system access, it is important to address both the applications for which sUAS can be used as well as the potential users of these systems and their impacts on the using communities. Broadly speaking, these applications and user communities can be broken into two main application objectives, namely, those being used for commercial purposes and those being used in the service of the "public good". (Note

\footnotetext{
${ }^{1}$ Head, Small Unmanned Aerial Vehicle Laboratory (SUAVELab), NASA Langley Research Center, Senior Member AIAA.

${ }^{2}$ Insert Job Title, Department Name, Address/Mail Stop, and AIAA Member Grade for third author.

${ }^{3}$ Insert Job Title, Department Name, Address/Mail Stop, and AIAA Member Grade for fourth author (etc).
} 
that there can be some overlap between applications when, for example, a regulatory requirement of a public agency is carried out by commercial firms.)

\section{A. Commercial Applications}

For the potential commercial uses of sUAS, it is easiest to target those applications for which there has already been a demand expressed. These include:

- Aerial photography and GIS

- Agriculture and crop surveys

- Outdoor advertising and banner towing

- Security services

- "Fly by" services (meter reading, aerial inventory, etc.)

- Entertainment and recreation

- Research and Development

- Fish spotting

- Training

Once airspace access has been opened to commercial users, it is likely that other applications will appear which have not been considered.

\section{B. Public Use applications}

In terms of sUAS used in the service of the "public good", there are again several applications for which there has already been an expressed demand. These public use aircraft currently must obtain a Certificate of Authorization from the FAA in order to operate as a public UAS. In terms of simple present demand, the FAA has indicated that $80 \%$ of the active CoAs for 2011 are for sUAS. The types of public operations which could be of great value include:

- Law enforcement (Federal, state, and local)

- Support to first responders (firefighters, HAZMAT)

- Traffic monitoring

- Pipeline and infrastructure monitoring (which may be conducted by commercial firms)

- Disaster response

- Weather observation and Earth Science

- Environmental monitoring (air quality, runoff, etc.)

- Regulatory and taxation

- Research and Education

- Wildlife management

- Wildfire detection and support

- Department of Defense (DoD)

\section{Economic Impact}

The economic impacts of sUAS usage can be categorized into four bins, the gross business base and/or employment from the users conducting such operations, the business base and/or jobs created by the suppliers of the systems to end users, the increase in GDP and employment associated with any net improvement in existing production or business base that can be reasonably attributed to use of sUAS, and a net increase in GDP or employment from totally new businesses that are created as a result of sUAS access to the NAS. Note that for a given commercial application, there may be some from all four bins whereas for public applications there may be fewer areas of increased economic impact.

\section{A. Commercial Applications}

The economic impact of sUAS operations from commercial enterprises can be estimated by looking at the potential user base and estimating what level of activity may result from their use of sUAS. For example, the Department of Agriculture, in its 2007 Census of Agriculture data, indicates that there are approximately 1.3million harvested cropland farms (out of 2.2million total farms) comprising some 310 million acres. These farms would be the most likely candidates to use sUAS systems to improve crop yields. Part of the attraction for sUAS to perform this function is that roughly half (46.3\%) of the 1.3 million farms are 200 acres or less in size. These farms are 
unlikely to be able to afford larger systems, even if used in aggregate or a timesharing arrangement. If just $10 \%$ of the 1.3 million farms begin to use sUAS systems, this would create a need for roughly 26,000 sUAS systems per year. This would create roughly 6500 jobs for sUAS manufacturing and as many as 2600 jobs in "for-hire" sUAS operations. This could translate to a roughly half a billion dollar industry segment. In addition, if the use of sUAS could only provide a $1 \%$ improvement in across-the-board crop yield improvement nationwide, this would translate into a \$1.43billion increase in the value of the crops sold. Note that from a regulatory standpoint, smaller farms could use low altitude, relatively short endurance platforms to make use of the technology whereas larger farms would need to be able to fly higher, faster, and beyond line-of-sight to take similar advantage. Although hard to quantify the benefits, potential reductions in the use of fertilizers, pesticides, etc. by the use of small UAS could provide a vast improvement in the local ecosystems of many of the farms which take advantage of the technology. Compiling the impacts from the other sUAS applications listed above yields similarly large results.

\section{B. Public Use Applications}

Similarly, the public use applications are also numerous. Looking at just one example, there are approximately 19,000 independent Federal, state, and local law enforcement agencies located throughout the US. According to the Us Department of Justice's 2007 Census of Law Enforcement Aviation Units (CLEAU), of 941 "large" agencies (over 100 sworn officers) surveyed, only 21\% currently have a manned aviation unit. The median expenditure for these aviation units was roughly $\$ 1.5$ million. In addition, the average cost per flight hour for maintenance and fuel was $\$ 99$ for fixed-wing and \$242 for helicopters. In considering what the potential usage for law enforcement could be, one recent survey indicated that roughly half of the 19,000 agencies are currently considering the use of sUAS when the regulations permit widespread use. Assuming that $75 \%$ of the "large" agencies and only $25 \%$ of the "small" agencies opt to use sUAS, this would mean approximately 4200 units per year would be needed. It is likely that many "operators" would be police officers cross trained in sUAS operations and thus the number of "new" operator jobs would likely be roughly 2000 nationwide. There are also some operations which, while "public" may actually be conducted by private firms such as pipeline monitoring. There are currently some 880,000 miles of drinking water pipelines in the US, according to the EPA. In 2009, the American Society of Civil Engineers estimated that these pipelines lost some 7 billion gallons of water per day compared to 43 billion gallons per day actually used or roughly $15 \%$. If the water loss percentage could be reduced by a mere $1 \%$ using sUAS to reduce leak detection times, this would reduce lost water expenditures by some \$2.5billion. Surveying the pipelines which are above ground and/or with leak detection easily visible would likely require some 500 sUAS platforms for a once-per-day search. According to the Pipeline and Hazardous Materials Safety Administration (PHMSA) data, some 3000 companies operate 175,000 miles of onshore and offshore Hazardous Liquid pipelines; 321,000 miles of onshore and offshore Gas Transmission and Gathering pipelines and 2,066,000 miles of Natural Gas Distribution mains and service pipelines. Although these pipelines must be inspected regularly, PHMSA only has 113 full-time pipeline inspectors and relies heavily on state inspectors and the companies operating the pipelines themselves to maintain their safety. Pipeline monitoring is often quoted as an obvious use for UAS. For gas and hazardous liquid pipelines, leak detection via UAS would need to be done at very low altitude ( 200ft) for direct, in-situ measurements to be effective. Even "remote" sensing for the types of materials being carried are most effective at less than $1000 \mathrm{ft}$. Monitoring these pipelines would require some 1500 sUAS units just to cover the vast distances required. As a result, there would be a need for some 400 units per year and nearly 2000 jobs created for operations.

Other local public users include between 26,000 and 30,000 independent fire departments in the US staffed by both professional and volunteer firefighters. Note that roughly $70 \%$ of these fire departments are all volunteer. Typically, when a fire department responds to a fire, there is a period of time required to conduct a situation assessment of the nature and extent of the fire as well as develop a plan of attack. It is extremely rare that these firefighters have any type of "birds eye view" camera or infra-red measurements to help with this assessment. In some cases, this lack of situational awareness has directly contributed to the deaths of firefighters, such as the deaths of two firefighters in Chesapeake, Virginia. Having a sUAS available to provide real-time video for the 480,000 structure fires in the US every year would provide a much-needed safety improvement. Similarly, having better direct information would likely reduce the damage to structures owing to a shorter evaluation time and better allocation of firefighting resources. If only a $1 \%$ reduction in damage due to fire occurs, this results in a loss savings of \$108million per year. While most of the fire departments that serve large metropolitan areas would likely take advantage of the technology, smaller departments may not be able to do so. Nonetheless, it is not unreasonable to consider that if $25 \%$ of the smaller departments and $50 \%$ of the larger departments make use of sUAS, this would result in sales of roughly 3000 units per year and a total job creation of roughly 2100. 


\section{Economic Impact Summary}

In summary, the economic benefit from relatively unrestricted sUAS operations is substantial. The total economic impact from unrestricted sUAS NAS access could be over \$8billion per year with a net job creation of over 34,000. In addition, the societal benefits from improving the environment and our understanding thereof, supporting law enforcement, first responders, and other public agencies as well as promoting aerospace education is profound.

\section{Operational Framework}

\section{A. Operational Tempo}

In terms of usage, the previous section detailed the general types of application demand. From this, it is reasonable to conclude that there are applications for sUAS in remote, rural, suburban, and urban areas. Using the application demand figures, if each potential platform averages one use per day, it is possible that sUAS operations could well exceed 200,000 per day throughout the country or over 75 million operations per year.

In addition, the DoD has indicated in their NAS Integration report for 2010 that there are presently 146 UAS personnel units at 63 locations throughout the US. By 2015, the DoD expects to have 197 UAS units at 105 locations needing some 275,000 flight hours per year in the NAS for training and proficiency/currency. Although this number includes all classes of UAS, the Army has indicated that of the slightly more than 1,000,000 UAS hours since 2001, over 300,000 were for sUAS. This would indicate that some 82,000 flight hours or 164,000 operations per year would be conducted by DoD using sUAS.

The FAA has indicated that of the currently active public operator CoAs issued, some $80 \%$ are for sUAS operations. This would indicate a clear bias on the part of public (Federal, state, and local) agencies to use sUAS versus their larger counterparts.

Clearly, the level of NAS access granted to sUAS will determine both the level of utilization and the impact on the NAS itself. This level of access consists not only of the geospatial constraints (where and at what altitudes) but also what equipage will be required, if any.

\section{B. Regulatory Framework}

The current rules for NAS access comprise both "airworthiness" (e.g. 14 CFR 23, 25 for aircraft) as well as "operations" (e.g. 14 CFR 91 and others). However, in considering what rules may or may not be applicable to sUAS it is instructive to keep in mind that the current regulations are predicated on two basic premises: First, that aircraft should be made safe for their occupants and Second, that aircraft should be operated so as to keep them from colliding with one another to prevent harm to both sets of occupants. With sUAS, there are no occupants. Therefore the primary focus of much of the highly detailed proscriptive nature of many of the regulations is irrelevant or not applicable to sUAS.

However, taken as a whole, the current regulations can provide some insight into a "level of risk" that is already assumed by current NAS users that could be utilized as a potential framework for sUAS inclusion. Another potential issue not generally addressed by current regulations is the potential risk to ground personnel and property. This would clearly be more important to sUAS since many of the applications being considered involve low altitude operations in and around urban areas.

One consideration that often creates exceptional debate is the question of "How 'small' is small?". Rather than attempt to create a definition directly, it might be possible to create some categories of "small" based upon certain risk levels already in the current regulations.

First, it is reasonable to postulate that there exists a size of sUAS that is so small that it presents almost no potential risk or hazard to either other aircraft or ground personnel no matter where it operates or who operates it. There is presently no data that would definitively identify the upper limit of what this size, weight, and/or kinetic energy might be. Testing sUAS of different types colliding with aircraft components, ground "targets" and instrumented anthropomorphic dummies would provide this limit.

The next potential category involves looking at existing regulations for potential insight on current collision risk hazard abatement. The existing Part 23 regulation calls for a bird-strike windshield protection requirement of $2 \mathrm{lb}$. The existing Part 25 regulations call for bird strike resistance of $4 \mathrm{lb}$. to the windshield, 8lb. to the engine and airframe. Further, Part 101 defines a "free balloon" and indicates:

“(4) Except as provided for in §101.7, any unmanned free balloon that-

$$
4
$$

American Institute of Aeronautics and Astronautics 
(i) Carries a payload package that weighs more than four pounds and has a weight/size ratio of more than three ounces per square inch on any surface of the package, determined by dividing the total weight in ounces of the payload package by the area in square inches of its smallest surface;

(ii) Carries a payload package that weighs more than six pounds;

(iii) Carries a payload, of two or more packages, that weighs more than 12 pounds; or

(iv) Uses a rope or other device for suspension of the payload that requires an impact force of more than 50 pounds to separate the suspended payload from the balloon.”

These existing rules define a "level of hazard" that is associated with birds and free balloons in terms of a collision hazard that is currently an "assumed risk" for users of the NAS. It is possible to use similar verbiage to construct a sUAS rule that would allow sUAS to be similar in weight and density to the existing free balloon payloads, i.e., $12 \mathrm{lb}$. total, no single component more than $6 \mathrm{lb}$. and no component more dense than 3oz./in^2. This would provide an adequate size for a large variety of small UAS operations described in earlier sections.

Part 101 also proscribes certain limits on the locations from which free balloons may operate and other operational limitations. However, many of these operational limitations are due to the lack of maneuverability and control of the balloon's trajectory, which would not be an issue with sUAS. If such characteristics were taken into consideration, it is likely that this size sUAS could operate in large classes of airspace without equipage or other constraints as they would pose no more of a hazard than free balloon payloads. In addition, since birds typically are unpredictable, they represent more of a hazard to current NAS users than sUAS operations as sUAS operations would be controlled either autonomously or via human operator.

The FAA issued an Advisory Circular, AC-91-57, that has been used as the basis for operations of many "model aircraft" operations since 1981. This voluntary compliance document suggests that model aircraft operations restrict their altitudes to $400 \mathrm{ft}$. or less, and if within 3 miles of an airport, to notify the airport operator or cognizant ATC facility. Note that AC 91-57 does not have any size limitation specifically called for. Many current model aircraft operations regularly exceed 400ft. AGL and many model aircraft clubs operate at airports/airfields and have done so safely for decades. One of the most prominent model aircraft organizations, the Academy of Model Aeronautics (AMA), has a member safety code. This code calls for models to generally be 55lb. or less, although a "waiver" process exists to allow models up to 100lb., to be flown at AMA sanctioned airfields and events. Furthermore, the AMA also has an active "turbine waiver" program that provides turbine powered model aircraft, some of which can fly up to 200kts., to also operate under AMA rules and insurance coverage. However, there is presently no published data that indicates that operating under such a safety code can be directly attributed to any specific increase in safety nor is there any data to support a lack of such a safety code would cause a reduction in net safety. Furthermore, there is a general lack of data concerning the reliability of COTS systems and subsystems that could be used in a safety hazard risk analysis. This includes COTS autopilot reliability and navigational accuracy, command and control link reliability, particularly in urban areas when using unlicensed bands, servoactuator reliability, motor and battery reliability, video link stability, ground station reliability, many of which are Windows based computers, systemlevel reliability, and others.

All present model, sUAS hobbyists, and "toy" aircraft flyers yield right-of-way to manned aircraft and suspend operations when manned aircraft are in the vicinity. This presents a rulemaking dilemma. Generally, in the manned aircraft rules, slower, less maneuverable aircraft have the right of way over faster. Thus, gliders must yield to balloons, powered aircraft must yield to gliders, faster aircraft must yield to slower, etc. The sUAS interaction with manned aircraft whereby sUAS yield to manned aircraft is a convention not due to a specific rule requirement (although it is suggested in AC 91-57) but reflects the reality that it is far easier for a ground based operator to see and avoid a large manned aircraft than it is for a manned aircraft to see and avoid a sUAS. Specific experiments need to be conducted to determine the ability of manned aircraft pilots to detect and discern the intent of sUAS under a variety of conditions. Only when such ability is quantified can a rule and its impact on other NAS users' ability to see-and-avoid be assessed to any significant degree. This is particularly true when the "avoid" part of the rule may differ from existing convention, such as would be the case with sUAS yielding to manned aircraft.

Attempting to discern an "upper limit" on what defines a "small" UAS is also difficult. Current NAS users include Part 103 Ultralight operations. For these operations, empty weight is generally limited to 254lb. With a pilot and fuel, this represents a vehicle of some 500lb. in weight that can be travelling up to 60kt. airspeed. Note that there are relatively few restrictions on where ultralight aircraft may operate since they may operate in virtually any airspace class "with permission" and are specifically excluded from many of the rules constraining Part 91 operations. Typically, these operations are conducted during daylight (or civil twilight if equipped with anticollision lighting) and may not be operated over ill-defined "congested areas". In addition, there are no requirements for airworthiness, registration, or pilot aeronautical knowledge. Clearly, this represents the "upper limit" of the current risk/hazard in terms of vehicle size and kinetic energy. It also helps define at least one boundary, by 
exclusion, of what size potentially non-airworthy vehicle should NOT be allowed to fly directly over people (since flight over open-air assemblies are prohibited under Part 103). Note also that for experimental certificated aircraft, there are relaxed restrictions once a certain number of hours of successful flight have been demonstrated by the aircraft. It is reasonable to consider that some form of "proof time" might be appropriate for larger sUAS that wish to fly over populated areas. As a "reinforcement" to this upper limit possibility, some foreign aviation authorities are considering 150kg. (330lb.) as an upper limit to what is considered "small". It is clear that an arbitrary limitation for what defines the upper limit of small is neither desirable nor defensible.

\section{Operational Framework}

It is conceivable that there would be varying levels of access for sUAS based upon credible risk assessments and mitigations of those risks. For example, a property owner should be allowed to operate a "small" UAS over their own property up to an altitude of 500ft. This would be consistent with allowing the operator, such as a small farm, to assume an "acceptable" risk if they so choose. Also, since manned aircraft are constrained by regulation to maintain a minimum altitude above people and structures, there would be very little likelyhood of collision with other NAS users. Similarly, in urban areas, small UAS could operate at or below the surrounding building heights (or up to 250ft. above - similar to tethered balloons) without likelihood that a manned aircraft would be present. Model aircraft and hobbyist operations would continue as they are currently conducted with the same suggested 400ft. operating altitude (with higher altitudes allowed under controlled conditions). Operators of sUAS under these circumstances would not be required to undergo the same medical and testing that manned aircraft pilots currently do.

For other operations, risk could be mitigated by a variety of means. Primarily, those sUAS whose size and weight make them inherently safe should be allowed unrestricted access to the NAS. As the size and kinetic energy increases, there would need to be a corresponding increase in the control reliability to ensure that the vehicle is under positive control at all times (either performing its mission, performing a flight termination, or navigating directly to a pre-determined safe rally point). Exactly what these risk levels are and what risk mitigations can be applied needs to be the subject of a vigorous research effort.

Equippage would also need some investigation. For most operations of sUAS, there would be little benefit to the requirement for equipment such as ADS-B or a sense-and-avoid system. However, in the case of using sUAS for higher altitude operations, such equipage may be necessary to enter certain classes of airspace.

\section{Conclusions}

Small UAS have a broad base of both commercial and public use applications. If relatively unrestricted access to the NAS is granted, the potential economic impacts could be enormous. Further, it is likely that many sUAS operations can be conducted in a safe and effective manner with no regulation. Other operations should only be regulated to the level of risk they pose to other NAS users and ground personnel and property. 